\title{
Editor's Remarks
}

This issue of the Canadian Journal of Program Evaluation (CJPE) is one of our most comprehensive to date. Not only does it include five full articles, five practice notes, and two book reviews, but it also covers a wide range of evaluation-related topics, practices, and studies. I am pleased to note that our editorial team continues to receive high-quality submissions, and I encourage you to keep thinking of the CJPE as an outlet for your work.

The articles and practice notes included in this issue focus on four recurring themes that reflect current topics in our field. First, evaluative thinking and capacity building in non-governmental organizations is the subject of articles by Rogers, Kelly, and McCoy, as well as by Lu, Elliot, and Perlman. Both articles provide insights into the facilitators of, and barriers to, evaluation capacity building as well as the multiple roles played by evaluators in fostering evaluative thinking amongst organizational staff members. Second, process evaluation appears to be of interest to many evaluators and researchers: Leblanc, Gervais, Dubeau and Delame focus on process evaluation for mental health initiatives, while Parrott and Carman provide an example of how process evaluation can contribute to program scaling-up efforts. Chechak, Dunlop, and Holosko also focus on process evaluation and its utility in evaluating youth drop-in programs. Teachers and students of evaluation may be interested in our third theme, which focuses on student contributions to evaluation, both through peer-mentoring-as described in the practice note written by LaChenaye, Boyce, Van Draanen, and Everett-and through the CES Student Evaluation Case Competition-described in a practice note written by Sheppard, Baker, Lolic, Soni, and Courtney. And fourth, we continue to advance our methodological approaches to evaluation, and this is reflected in an article on evaluation in Indigenous contexts by Chandna, Vine, Snelling, Harris, Smylie, and Manson, as well as in an article on the use of an outcome monitoring tool for performance measurement in a clinical psychology setting by Rosval, Yamin, Jamshidi, and Aubry. Czechowski, Sylvestre, and Moreau also feature methods in their practice note on secure data handling for evaluators, a key competency that continues to evolve as our data collection and storage mechanisms adapt to new technology.

In addition to these articles and practice notes, this issue also features two book reviews that are sure to interest our readers. First, Bhawra provides an account of Developing Monitoring and Evaluation Frameworks, by Patrick Markiewicz (2016), and, second, Sellick reviews Collaborative, Participatory, and Empowerment Evaluation: Stakeholder Involvement Approaches, by David Fetterman, Liliana Rodriguez-Campos, Ann Zukoski, and other contributors (2018). 
On behalf of the entire editorial team, I hope that these papers stimulate discussion and reflection and support the advancement of our collective knowledge and practice. As always, if you have feedback on this issue, please contact me. I would love to hear your thoughts!

Isabelle Bourgeois Editor-in-Chief 